\title{
Fragile X mental retardation protein regulates skeletal muscle stem cell activity by regulating the stability of Myf5 mRNA
}

\author{
Ryo Fujita ${ }^{1,2+}$, Victoria Zismanov ${ }^{1,2+}$, Jean-Marie Jacob ${ }^{1,2,4}$, Solène Jamet ${ }^{1}$, Krum Asiev ${ }^{1,3}$ and Colin Crist ${ }^{1,2^{*}}$ (D)
}

\begin{abstract}
Background: Regeneration of adult tissues relies on adult stem cells that are primed to enter a differentiation program, while typically remaining quiescent. In mouse skeletal muscle, these features are reconciled by multiple translational control mechanisms that ensure primed muscle stem cells (MuSCs) are not activated. In quiescent MuSCs, this concept is illustrated by reversible microRNA silencing of Myf5 translation, mediated by microRNA-31 and fragile $X$ mental retardation protein (FMRP).
\end{abstract}

Methods: In this work, we take advantage of FMRP knockout $\left(\mathrm{Fmrl}^{-1-}\right)$ mice to support the role for FMRP in maintaining stem cell properties of the MuSC. We compare the activity of MuSCs in vivo after acute injury and engraftment, as well as ex vivo during culture. We use RNA immunoprecipitation and 3'UTR poly-adenine (poly(A)) length assays to assess the impact of FMRP on the stability of transcripts for myogenic regulatory factors.

Results: We show that RNA-binding FMRP is required to maintain the MuSC pool. More specifically, FMRP is required for stem cell properties of muscle stem cells, which include MuSC capacity to prime the myogenic program, their selfrenewal, and their capacity to efficiently regenerate muscle. We provide evidence that FMRP regulation of MuSC activity occurs in part by the capacity of FMRP to directly bind Myf5 transcripts and impact rates of Myf5 deadenylation. Conclusions: Our results provide further evidence supporting a role for post-transcriptional silencing platforms by RNAbinding proteins in maintaining stemness properties of adult stem cells. In addition, deregulated MuSC activity in the absence of Fmr1 may have implications for fragile X syndrome, which is associated with muscle hypotonia during infancy.

Keywords: Muscle stem cell, Satellite cell, Fragile X mental retardation protein, Myogenic regulatory factor Myf5

\section{Background}

Regeneration of adult tissues relies on the activity of adult stem cells that are primed to enter a tissue-specific differentiation program. In the skeletal muscle, these adult stem cell properties are illustrated by "satellite cells," named for their position underneath the basal lamina of myofibers [1]. Satellite cells express members of the paired homeodomain family of transcription factors, $P A X 7$, and in subset of muscle, $P A X 3[2,3]$. During development, PAX3/PAX7 are important regulators of

\footnotetext{
* Correspondence: colin.crist@mcgill.ca

${ }^{\dagger}$ Equal contributors

'Lady Davis Institute for Medical Research, Jewish General Hospital, Montreal, Quebec H3T 1E2, Canada

${ }^{2}$ Department of Human Genetics, McGill University, Montreal, Quebec H3A

1B1, Canada

Full list of author information is available at the end of the article
}

myogenic progenitor survival and are required to activate the expression of myogenic determination genes $M y f 5$ and $M y o D$, with consequent rapid muscle differentiation [4].

Quiescent satellite cells are notable for being primed to activate the myogenic program while remaining quiescent. These features are reconciled by translational control mechanisms regulating gene expression by which microRNAs and RNA-binding proteins prevent the translation of messenger RNAs (mRNAs) for myogenic regulatory factors $M y f 5$ and $M y o D[5,6]$, as well as cell proliferation regulators such as Dek [7]. Furthermore, some mRNAs, such as those for Myf5, accumulate in RNA granules present in the quiescent satellite cell, which serve as sites of mRNA storage. These RNA granules form in response to the phosphorylation of eIF $2 \alpha$, 
which is a translational control mechanism regulating global protein synthesis that is required to maintain satellite cell quiescence [8].

Translational control of gene expression is an important mechanism by which a cell rapidly responds to its environment. These responses can be mediated by the activity of RNA-binding proteins such as fragile $\mathrm{X}$ mental retardation protein (FMRP), which regulates mRNA translation, localization, and stability [9]. The precise mechanisms by which FMRP influences translation of bound mRNAs remain unclear, but its phosphorylation at serine residue 499 is correlated with its presence in stalled polyribosomes [10]. FMRP is also associated with the RNA-induced silencing complex (RISC), suggesting that FMRP inhibits translation, at least in part, in cooperation with the microRNA pathway [11-13]. Emerging evidence indicates that FMRP has bifunctional activity to either permit or prevent the translation of transcripts to which it binds. In hippocampal neurons, phosphorylated FMRP (P-FMRP) binds to the RISC complex associated with miR-125 to silence PSD-95 mRNA as it is transported to the termini of dendrites for localized translation. Translation of PSD-95 mRNA at dendritic spines requires the dephosphorylation of FMRP, which causes the dissociation of PSD-95 mRNA from miR-125/RISC silencing [14].

P-FMRP is also present in quiescent muscle stem cells, where we proposed that it facilitates the reversible inhibition of Myf5 translation by microRNA-31. Upon satellite cell activation, FMRP is dephosphorylated. Blocking the FMRP phosphatase PP2A with okadaic acid prevents the translation of accumulating Myf5 transcripts and delays the activation of the myogenic program [5].

In this study, we use $\mathrm{Fmrl}^{-1-}$ mice to further support a role for FMRP in the stem cell properties of the satellite cell. We propose a mechanism by which FMRP RNA binding activity promotes the stability of myogenic regulatory factors such as Myf5. In the absence of FMRP, satellite cells have reduced capacity to prime the myogenic program, rapidly activate, self-renew, and regenerate muscle.

\section{Methods}

\section{Mice}

Care and handling of animals were in accordance with the federal Health of Animals Act, as practiced by McGill University and the Lady Davis Institute for Medical Research. For muscle regeneration, 6- to 8-week-old mice were anesthetized by isoflurane (CDMV) inhalation and $50 \mu \mathrm{l}$ of $10 \mu \mathrm{M}$ cardiotoxin (ctx) (Sigma) was injected into the tibialis anterior (TA) muscle. At 21 days after injury, the muscles were harvested for analysis by immunofluorescence. Cell engraftment assays were performed as previously described [8]. Immunocompromised 8-week-old Foxn $1^{n u / n u}$ female mice (Jackson Laboratories) were used.
Donor cells were engrafted into the TA muscle, $24 \mathrm{~h}$ after the hindlimbs were exposed to 18 Gy irradiation.

\section{Cell and single-fiber isolation and culture}

Satellite cells were isolated from the abdominal and diaphragm muscle, or from the ctx-injured TA muscle, of 5- to 8-week-old $\mathrm{Pax}^{\mathrm{GFP} /+}$ and $\mathrm{Pax}^{\mathrm{GFP/+}}{ }^{\mathrm{G}} \mathrm{Fmrl}^{-/-}$ mice (Jackson Laboratories) [15] as previously described [16] using a FACSArialII cell sorter (BD Biosciences) or with magnetic beads (MACS Satellite Cell Isolation Kit, together with anti-Integrin a-7 MicroBeads, Miltenyl Biotec). Isolated cells were cultured in 39\% DMEM, 39\% F12, 20\% fetal calf serum (Life Technologies), and 2\% UltroserG (Pall Life Sciences). Single fibers were isolated by trituration of $0.2 \%$ collagenase D (Sigma)-treated $e x$ tensor digitorum longus (EDL) muscle of adult mice [5].

\section{Immunodetection}

Immunofluorescence labeling of cultured satellite cells, single EDL myofibers, and transverse sections of TA muscle was performed as described previously [5, 8]. For immunolabeling with antibodies against GFP, TAs were fixed for $2 \mathrm{~h}$ in $0.5 \%$ paraformaldehyde at $4{ }^{\circ} \mathrm{C}$ and equilibrated overnight in $20 \%$ sucrose at $4{ }^{\circ} \mathrm{C}$. Tissues were mounted in Frozen Section Compound (VWR) and flash frozen in a liquid nitrogen cooled isopentane bath. For immunoblotting, cell lysates were prepared as described previously [5]. Densitometry of immunoblots was performed with ImageJ.

Primary antibodies were against PAX7 (DSHB, Pax7-c), MYF5 (Santa Cruz, sc-302), MYOD (SantaCruz, sc-304), LAMININ (Sigma, L9393), embryonic MHC (DSHB, F1.652), and $\beta$-ACTIN (Sigma, A5441). Alexa Fluor-488 and Alexa Fluor-594 conjugated secondary anti-mouse or anti-rabbit antibodies (Life Technologies) were used for immunofluorescence. Neuromuscular junctions were labeled with Alexa Fluor-488 bungarotoxin (Life Technologies). 5-Ethynyl-2'-deoxyuridine (EdU) (Life Technologies) was administered by a single intraperitoneal injection $(40 \mathrm{mg} / \mathrm{kg}$ ). After $24 \mathrm{~h}$, transverse sections of frozen TA muscle were fixed with $4 \%$ paraformaldehyde for $15 \mathrm{~min}$ and washed twice with $3 \%$ bovine serum albumin in PBS and permeabilized with $0.5 \%$ Triton in PBS. Staining was performed with the Click-it EdU Alexa Fluor 594 kit (Life Technologies) [17]. Images were acquired with an AxioImager M1 fluorescence microscope (Zeiss). Horseradish peroxidase (HRP) conjugated goat anti-mouse or antirabbit secondary antibodies (Jackson Immunoresearch) were used with the ECL Prime Western Blotting Detection reagents (GE Healthcare) to image immunoblots with ImageQuant LAS 4000 (GE Healthcare).

\section{RNA immunoprecipitation}

To immunoprecipitate FMRP mRNA protein complexes, $5 \times 10^{5} \mathrm{C} 2 \mathrm{C} 12$ cells were seeded in $10 \mathrm{~cm}$ plates. 
Twenty-four hours later, cells were transfected with $5 \mu \mathrm{g}$ pCAG-GFP [18] (gift from Connie Cepko, Addgene \#11150) (control) or pFRT-TODestFLAGHAhFMRP [19] (FLAG-hFMRP) (gift from Thomas Tuschl, Addgene \#48690) using jetPRIME transfection reagent (Polyplus tranfection) according to manufacturer's instructions. Twenty-four hours after transfection, cells were lysed with polysome lysis buffer. Lysate preparation and immunoprecipitation was performed as described [20] using antibodies against FLAG M2 (Sigma, F1804) or GFP (DSHB, 8H11) with the exception that after washes, the total immunoprecipitated RNA was isolated using TRIzol reagent, as described below.

\section{RNA analysis}

RNA was isolated from satellite cells or after immunoprecipitation of FMRP from $\mathrm{C} 2 \mathrm{C} 12$ cells with TRIzol reagent (Life Technologies) and treated with DNase (Roche). RNA was reverse-transcribed with Superscript III reverse transcriptase (Life Technologies). Primers for quantitative PCR were Pax7, forward 5'-CTCAGTGA GTTCGATTAGCCG-3', reverse 5' -AGACGGTTCCCT TTGTCGC-3'; Myf5, forward 5' -CTGTCTGGTCCCGA AAGAAC-3', reverse 5' -AAGCAATCCAAGCTGGAC AC-3'; $M y o D$, forward 5'-CCCCGGCGGCAGAATGGC TACG-3', reverse 5' -GGTCTGGGTTCCCTGTTCTGT GT-3', Myog, forward 5'-CAACCAGGAGGAGCGCG ATCTCCG-3', reverse 5' ${ }^{\prime}$-AGGCGCTGT GGGAGTT GCATTCACT-3', Myh3, forward 5'-GCATAGCTGC ACCTTTCCTC-3', reverse 5'-GGCCATGTCCTCAA TCTTGT-3', and Actb forward 5'-AAACATCCCCC AAAGTTCTAC-3' reverse 5'-GAGGGACTTCCTGT AACCACT-3'.

Levels of mRNA were measured using SYBR Green on a 7500 Fast Real Time PCR System (Applied Biosystems). Poly-adenine (polyA) length assays were performed with the Poly(A) Tail-Length Assay Kit (Affymetrix) using gene-specific primers for Myf5, 5-CAGCTTTGACA GCATCTAC-3' and 5'-TAGATAAGTCTGGAGCTGG A-3'; Pax7, 5'-GACTCCATCAAGCCAGGAG-3'; and 5'-TAGTAGGCTTGTCCCGTTTC-3'.

\section{Statistical analysis}

Graphical analysis is presented as mean \pm SEM. At least three independent replicates of each experiment were performed. Significance was calculated by unpaired Student's $t$ tests with two-tailed $p$ values: ${ }^{*} p<0.05$, ${ }^{* * *} p<0.01, * * * 0.001$.

\section{Results}

Fmr1 is required for the homeostasis of primed satellite cells To assess the impact of FMRP on the myogenic program, we began by examining satellite cells on single EDL myofibers isolated from wild-type and $\mathrm{Fmrl}^{-/-}$ mice by immunostaining for PAX7. On average, three satellite cells are present for each EDL myofiber isolated from $\mathrm{Fmrl}^{-/-}$mice, compared to the normal complement of six satellite cells per EDL myofiber isolated from wild-type mice (Fig. 1a, b). While satellite cells are spread evenly throughout the myofiber, they are also often found in proximity to neuromuscular junctions (NMJs) [21, 22]. Furthermore, denervation leads to activation of satellite cells throughout the skeletal muscle [23]. In Drosophila melanogaster, knockout of dFmr1 results in NMJs with altered structure and function [24]. We therefore asked whether the NMJ component of the satellite cell niche is altered in $\mathrm{Fmrl}^{-/-}$ mice. Using bungarotoxin labeling, we show that single EDL myofibers from both wild-type and $\mathrm{Fmrl}^{-/-}$mice have a single NMJ, often in proximity to satellite cells. Abnormal NMJ morphologies were not observed (Fig. 1c, d).

Next, we asked whether intrinsic defects exist in satellite cells isolated from $\mathrm{Fmrl}^{-/-}$mice. We examined transcript levels for $\operatorname{Pax} 7, M y f 5$, and $M y o D$ in satellite cells isolated from the muscle of adult wild-type and $\mathrm{Fmrl}^{-/-}$mice. In the absence of FMRP, we show reduced levels of Pax7, Myf5, and MyoD (Fig. 1e). While Myf5 and $M y o D$ transcripts are present in quiescent satellite cells, translational control mechanisms ensure MYF5 and MYOD protein do not accumulate [5, 6, 8]. The absence of FMRP does not increase numbers of satellite cells expressing MYF5 or MYOD on single EDL myofibers (Fig. 1f, g).

\section{Fmr1 is required for efficient satellite cell differentiation and self-renewal ex vivo}

Since lower levels of Myf5 and MyoD mRNAs are present in quiescent satellite cells isolated from the muscle of $\mathrm{Fmrl}^{-/-}$mice, we next asked if $\mathrm{Fmrl}^{-/-}$satellite cells are less primed to rapidly activate the myogenic program. Satellite cells on single EDL myofibers isolated from wild-type mice rapidly upregulate MYF5 and MYOD protein, within $1 \mathrm{~h}$, but this rapid upregulation is delayed in satellite cells on EDL myofibers isolated from $\mathrm{Fmr1}^{-/-}$mice (Fig. 2a). To examine the role of FMRP during satellite cell entry into the myogenic program, EDL myofibers derived from $w t$ and $\mathrm{Fmrl}^{-/-}$ mice were cultured for 2 days and immunostained with antibodies against PAX7 and MYOD. Numbers of satellite cells undergoing self-renewal (PAX7+MYOD-) are reduced on EDL myofibers isolated from $\mathrm{Fmrl}^{-1-}$ mice compared to those from wild-type mice, while those activating the myogenic program $(\mathrm{PAX} 7+\mathrm{MYOD}+)$ and undergoing differentiation (PAX7-MYOD+) are not significantly changed (Fig. 2b, c).

To examine later stages of differentiation, we cultured satellite cells isolated from the muscle of adult $\mathrm{Pax}^{\mathrm{GFP} /+}$ and $\mathrm{Pax} 3^{\mathrm{GFP} /+}$; Fmr1 $\mathrm{Fr-}^{-/}$mice for 4 days. Differentiating 

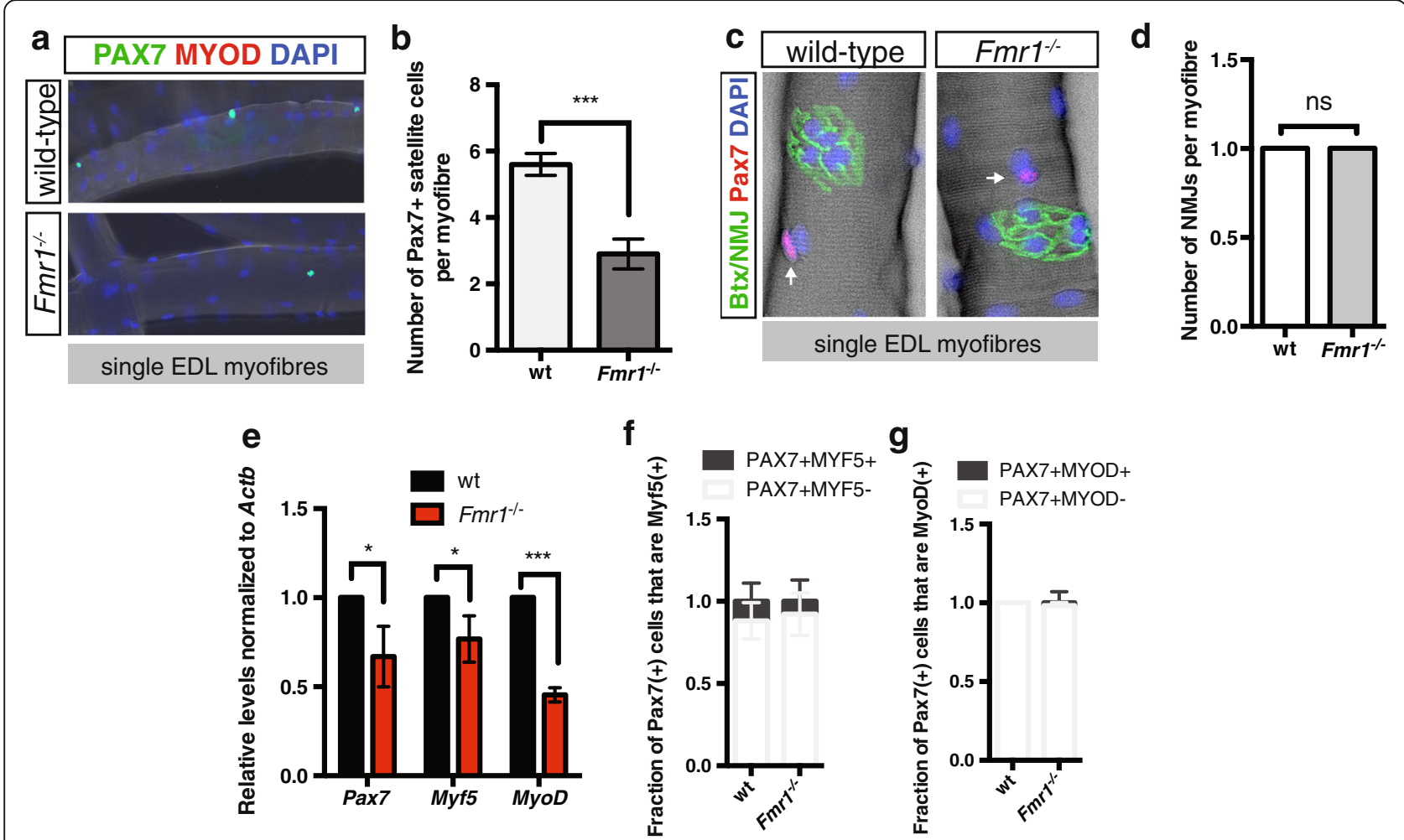

Fig. 1 Skeletal muscle of $\mathrm{Fmr}^{-/-}$mice have reduced numbers of satellite cells. a Immunostaining of PAX7 (green) on newly isolated single EDL myofibers isolated from adult wild-type (upper panel) and $\mathrm{Fmrl}^{-1-}$ (lower panel) mice. b Numbers of PAX7-positive satellite cells per EDL myofiber isolated from wild-type (white) and Fmr1 ${ }^{-1-}$ (gray) mice. c Immunostaining of PAX7 (red) and bungarotoxin (Btx) labeling of neuromuscular junctions (NMJ) on single EDL myofibers isolated from wild-type and $\mathrm{Fmr}^{-1-}$ mice. $\mathbf{d}$ Numbers of NMJs per EDL myofiber. e Levels of Pax7, Myf5, and MyoD transcripts, relative to Actb, in satellite cells isolated from adult muscle of wild-type (wt, black) and Fmr1 ${ }^{-1-}$ (red) mice. f Fraction of PAX7(+) satellite cells that immunostain for MYF5 on single EDL myofibers isolated from muscle of wild-type (wt) and Fmr1 ${ }^{-1-}$ mice. $\mathbf{g}$ Fraction of PAX7(+) satellite cells that immunostain for MYOD on single EDL myofibers isolated from muscle of wild-type (wt) and $\mathrm{Fmr}^{-1-}$ mice. All values indicate mean $(n \geq 3) \pm$ s.e.m. ${ }^{*} p<0.05$, ${ }^{* *} p<0.001$. ns not significant

satellite cells isolated from $\mathrm{Pax}^{\mathrm{GFP/+}}$; Fmr1 ${ }^{-/-}$mice have reduced expression of myogenic regulatory factor mRNAs MyoD and Myog, as well as myosin heavy chain Myh3 (Fig. 2d). The delay in differentiation of satellite cells isolated from $\mathrm{Pax}_{3}^{\mathrm{GFP} /+}$; $\mathrm{Fmrl}^{-/-}$mice is also evident by decreased levels of MYOG protein (Fig. 2e).

\section{Fmr1 is required for satellite cell activity to regenerate muscle and self-renew in vivo}

To ask whether defects observed in satellite cell self-renewal and differentiation are also observed in vivo, we next injured the TA muscle of wild-type and $\mathrm{Fmrl}^{-/-}$mice by intramuscular injection of cardiotoxin, a snake venom toxin that causes muscle fiber necrosis without affecting the viability of the satellite cell [25]. Two days after acute muscle injury by cardiotoxin injury in the TA muscle, satellite cells isolated from $\mathrm{Fmrl}^{-1-}$ mice show reduced levels of Pax7 and $M y f 5$ mRNAs, compared to those from wild-type mice (Fig. 2f). Satellite cells of $\mathrm{Fmrl}^{-/}$mice also exhibit reduced frequency of proliferation, compared to wild-type mice, as shown by decreased incorporation of EdU (Fig. 2g, h).
Ten days after muscle injury, we analyzed regeneration with antibodies against laminin and embryonic myosin heavy chain (embMHC). Evidence for a delay in muscle regeneration in the absence of Fmr1 is indicated by increased expression of embMHC and smaller myofibers (Fig. 3a, b).

Twenty-one days after muscle injury, we analyzed regeneration with antibodies against PAX7, embMHC, and lamina. At this time point, wild-type mice downregulate embMHC in favor of adult isoforms. $\mathrm{Fmrl}^{-/-}$mice continue to exhibit smaller myofibers with perduring expression of embMHC (Fig. 3c, d). We also observe reduced numbers of satellite cells returning to their normal position underneath the basal lamina of the myofiber in regenerating muscle of $\mathrm{Fmrl}^{-/-}$mice, compared to that of wild-type mice (Fig. 3e, f).

Although satellite cells in the muscle of $\mathrm{Fmrl}^{-/-}$ mice have reduced numbers after acute injury, the initial satellite cell pool is also reduced (Fig. 1a, b). We therefore asked if FMRP is required for satellite cell self-renewal by following their fate after engraftment. Satellite cell engraftment into the $D m d^{m d x}$ mouse 


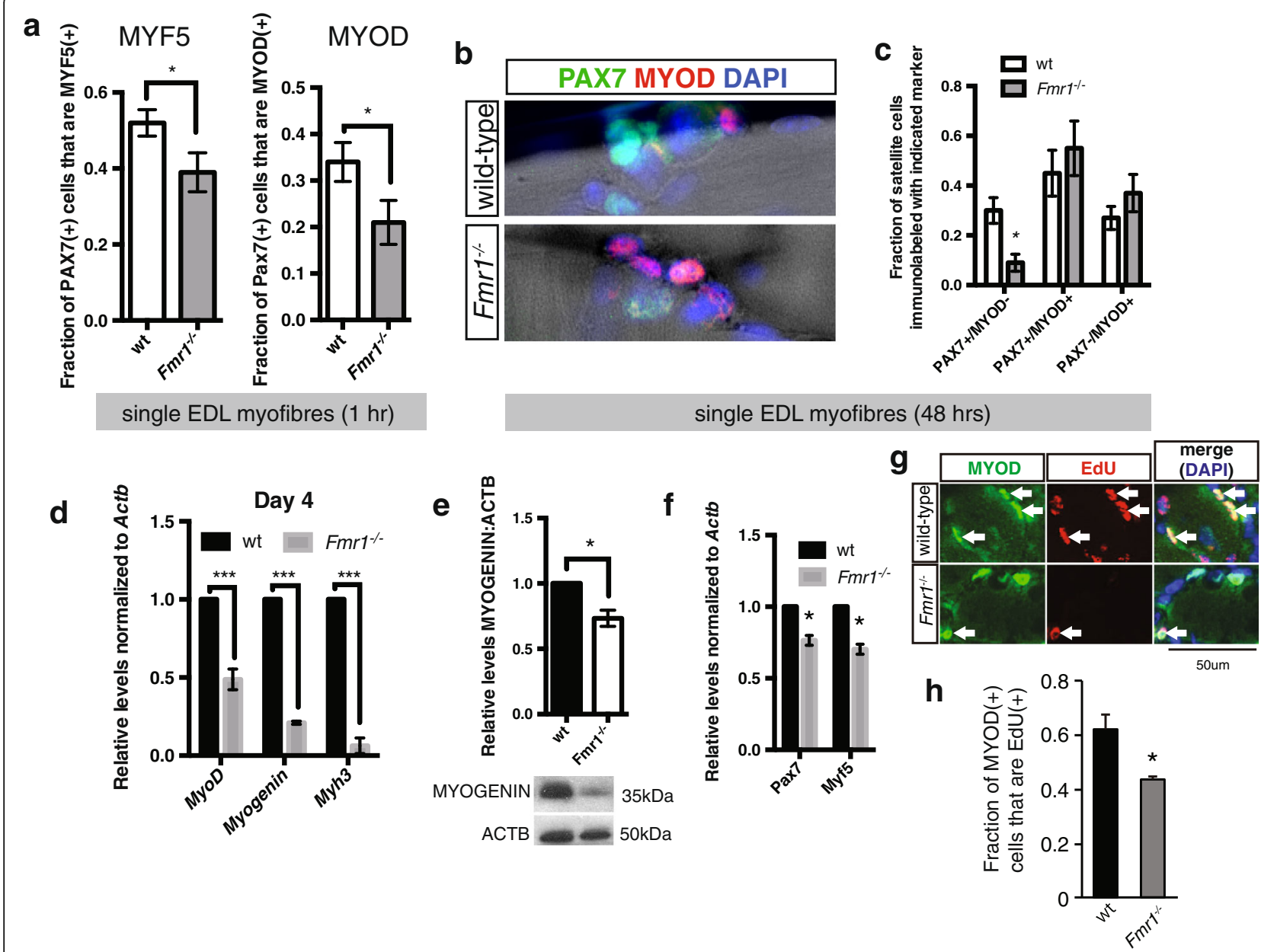

Fig. 2 FMRP is required for efficient priming of muscle stem cells to rapidly activate the myogenic program, self-renewal, and differentiation. a Fraction of PAX7(+) satellite cells accumulating MYF5 (left) and MYOD (right) after $1 \mathrm{~h}$ culture of single EDL myofibers. b Representative immunostaining of PAX7 (green) and MYOD (red) on single EDL myofibers isolated from adult wild-type and Fmr ${ }^{-1-}$ mice after $48 \mathrm{~h}$ culture. c Numbers of satellite cells undergoing self-renewal (PAX7+MYOD-), activating the myogenic program (PAX7+MYOD+) and differentiating (PAX7-MYOD+) on single EDL myofibers isolated from adult wild-type (white) and $\mathrm{Fmr}^{-1-}$ (gray) mice after 2-day culture. $\mathbf{d}$ Levels of MyoD, Myog, and Myh3 transcripts, relative to Actb, in satellite cells isolated from adult muscle of wild-type (wt, black) and $\mathrm{Fmr}^{-1-}$ (gray) mice, after 4-day culture. e Levels of MYOG protein, relative to ACTB, from cell lysates of 4-day cultured satellite cells isolated from muscle of wt (black) and $\mathrm{Fmr}^{-1-}$ (white) mice. Representative immunoblot is shown. MYOG levels are calculated by densitometry of three immunoblots. $\mathbf{f}$ Levels of Pax7 and Myf5 transcripts, relative to Actb, in satellite cells isolated from adult muscle of wild-type (wt, black) and $\mathrm{Fmr}^{-/-}$(gray) mice, 2 days after ctx injury. $\mathbf{g}$ Representative images of EdU(+) MyoD(+) satellite cells present on transverse sections of TA muscle, 3 days after ctx injury. White arrows indicate MYOD(+) EdU(+) satellite cells. Scale bar, $50 \mu \mathrm{m}$. $\mathbf{h}$ Fraction of MYOD(+) satellite cells that are $\mathrm{EdU}(+)$, as indicated in $\mathbf{g}$. All values indicate mean $(n \geq 3) \pm$ s.e.m. ${ }^{*} p<0.05,{ }^{* * *} p<0.001$

model of Duchenne muscular dystrophy permits the assessment of the intrinsic ability of satellite cells to differentiate and restore dystrophin expression, as well as self-renew to populate the engrafted muscle with a donor-derived satellite cell pool. We isolated satellite cells from $\mathrm{Pax} 3^{\mathrm{GFP} /+}$ and $\mathrm{Pax}^{\mathrm{GFP} /+}$; $\mathrm{Fmrl}^{-/-}$adult muscle and immediately engrafted 10,000 cells into the TA muscle of 18 Gy irradiated hindlimb of $D m d^{m d x}$; Foxn $1^{n u / n u}$ immunocompromised mice (Fig. 4a). Twenty-one days after engraftment, we show that satellite cells isolated from wild-type mice and $\mathrm{Fmrl}^{-/-}$mice have similar ability to contribute to hundreds of dystrophin-positive myofibers
(Fig. 4b, c). In contrast, satellite cells isolated from $\mathrm{Pax3}^{\mathrm{GFP} / \mathrm{H}}$; $\mathrm{Fmrl}^{-/-}$mice have reduced capacity to repopulate the satellite cell pool, determined by immunofluorescence with antibodies against PAX7 and GFP (Fig. 4d, e).

FMRP binds Myf5 mRNA and regulates poly(A) tail length Together, our data suggests that FMRP regulates the efficiency of (a) priming quiescent satellite cells such that they rapidly activate the myogenic program, (b) selfrenewal of satellite cells to maintain the satellite cell pool, and (c) differentiation of satellite cells to participate in 

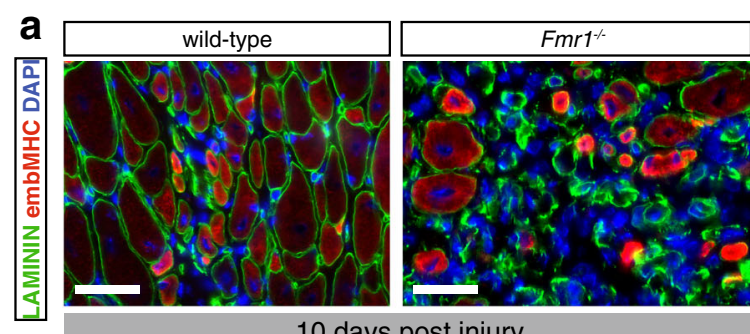

10 days post injury

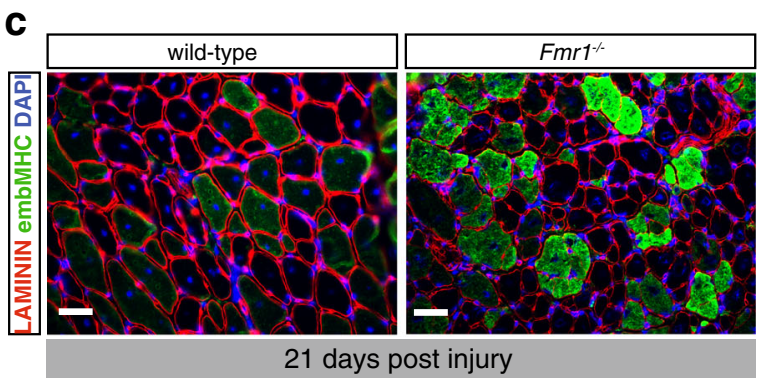

21 days post injury

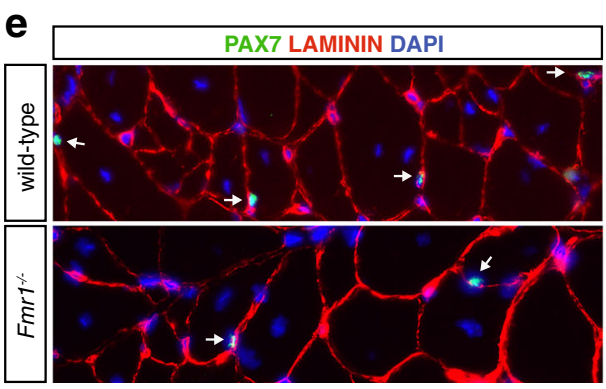

$\mathbf{f}$

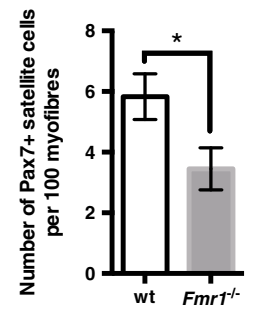

d

21 days post injury
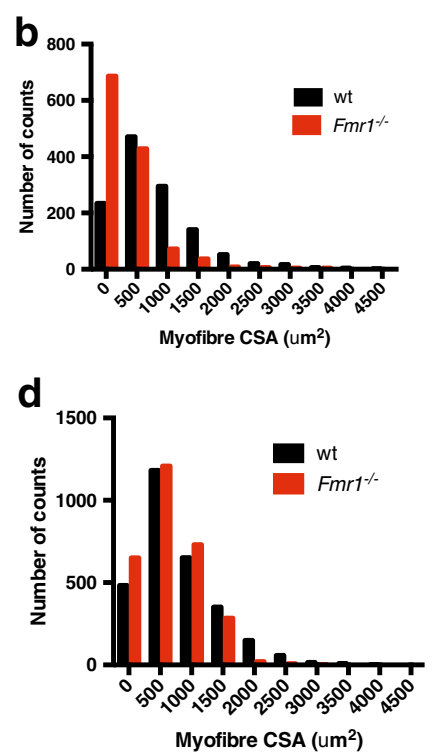

Myofibre CSA (um²)

Fig. 3 Delayed regeneration after acute injury of muscle in $\mathrm{Fmr}^{-1-}$ mice. a Immunostaining laminin (Lam, green), embryonic myosin heavy chain (embMHC, red) on transverse sections of TA muscle isolated from wild-type (left), and Fmr ${ }^{-1-}$ (right) mice 10 days after injury. Scale bar, white $50 \mu m$. b Mean myofiber cross-section area (CSA) of wild-type (white) and $\mathrm{Fmrl}^{-1-} \mathrm{TA}$ muscle 10 days after injury. Numbers of myofibers with greater area than the indicated bin label are shown. $\mathbf{c}$ Immunostaining laminin (Lam, red), embryonic myosin heavy chain (embMHC, green) on transverse sections of TA muscle isolated from wild-type (left), and $\mathrm{Fmrl}^{-1-}$ (right) mice 21 days after injury. Scale bar, white $50 \mu \mathrm{m}$. d Mean myofiber cross-section area (CSA) of wild-type (white) and $\mathrm{Fmrl}^{-1-}$ TA muscle 21 days after injury. Numbers of myofibers with greater area than the indicated bin label are shown. $\mathbf{e}$ Immunostaining PAX7 (green) laminin (red) on transverse sections of TA muscle isolated from wild-type (upper panel) or Fmr ${ }^{-/-}$(lower panel) 21 days after cardiotoxin injury. $\mathbf{f}$ Number of PAX7+ satellite cells present underneath the basal lamina, 21 days after injury of the TA muscle of wild-type (white) and $\mathrm{Fmrl}^{-1-}$ (gray) mice. Values indicate mean $(n \geq 3) \pm$ s.e.m. ${ }^{*} p<0.05$

regeneration of muscle. We therefore asked whether FMRP binds and regulates the stability of Myf5 transcripts. The G-quadruplex structure is the best characterized FMRP binding sequence [26], and more recently, two independent FMRP CLIP sequencing datasets have permitted a more comprehensive examination of FMRP binding consensus sites [27]. Myf5 mRNAs contain multiple FMRP consensus binding sites, which also assemble to form G-quadruplex structures (Fig. 5a). We therefore asked if FMRP directly binds Myf5 mRNA. We transfected $\mathrm{C} 2 \mathrm{C} 12$ cells with plasmids driving the expression of either GFP (control transfection) or FLAG epitope-tagged hFMRP [18] (FLAG-hFMRP) and subsequently performed RNA immunoprecipitation with antibodies against FLAG or GFP. Myf5 transcripts were enriched when lysates were immunoprecipitated with antibodies against FLAG, compared with antibodies against GFP (Fig. 5b). Pax7 transcripts are not enriched when lysates are immunoprecipitated with antibodies against FLAG or GFP (Fig. 5b).

If FMRP cooperates with RISC to reversibly repress Myf5 translation in quiescent satellite cells and PSD-95 in hippocampal neurons $[5,11,12,14]$, it remains unclear how excessive microRNA-dependent mRNA decay is avoided. Since RISC and RNA-binding protein-mediated silencing commonly occurs through the interaction with the CCR4/NOT1 deadenylase machinery $[28,29]$, we compared deadenylation of transcripts present in total RNA isolated from wild-type and $F m r 1^{-/-}$satellite cells after 3-day culture. Myf5 transcripts from satellite cells isolated from $\mathrm{Fmrl}^{-/-}$mice have shorter polyA tails than 


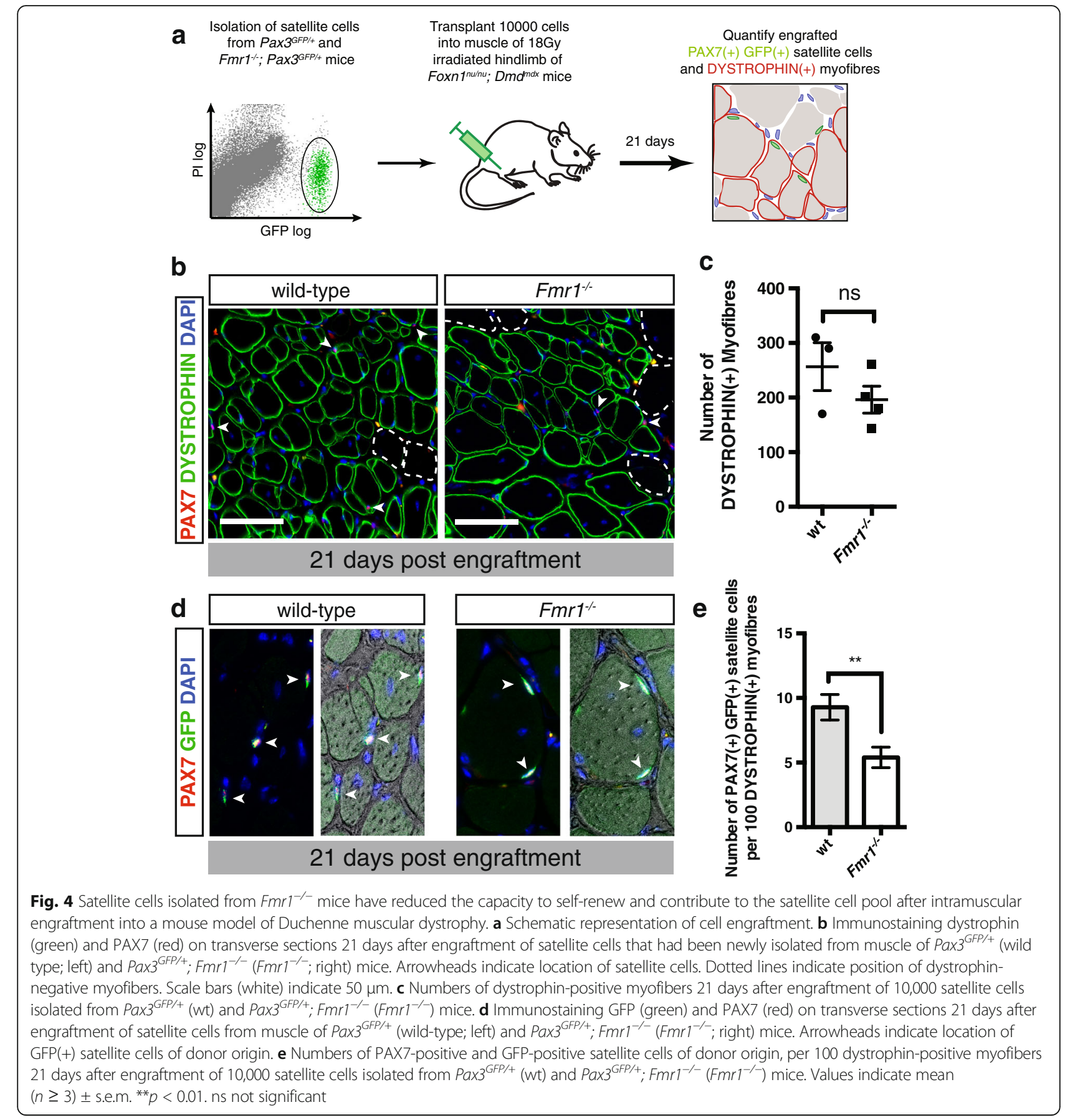

those isolated from wild-type mice. polyA tails of $\operatorname{Pax} 7$ transcripts remain largely unchanged (Fig. 5c). Therefore, FMRP negatively regulates $M y f 5$ mRNA deadenylation in satellite cells.

\section{Discussion}

Fragile $\mathrm{X}$ syndrome is the most commonly inherited form of intellectual disorder, affecting 1 in 1250 males and 2500 females [30]. Fragile X syndrome is a multiorgan disease that particularly leads to intellectual disorder, macroorchidism in males, and premature ovarian insufficiency in females. Fragile $\mathrm{X}$ syndrome is caused by the loss of function of Fmrl due to the increased expansion and hypermethylation of trinucleotide (CGG) repeats within the promoter of Fmr1 [31]. FMRP is an RNA-binding protein and is widely expressed in human tissues, but particularly abundant in neurons. The absence of FMRP in neurons is believed to cause translation dysregulation defects in mRNA transport that is required for localized protein 


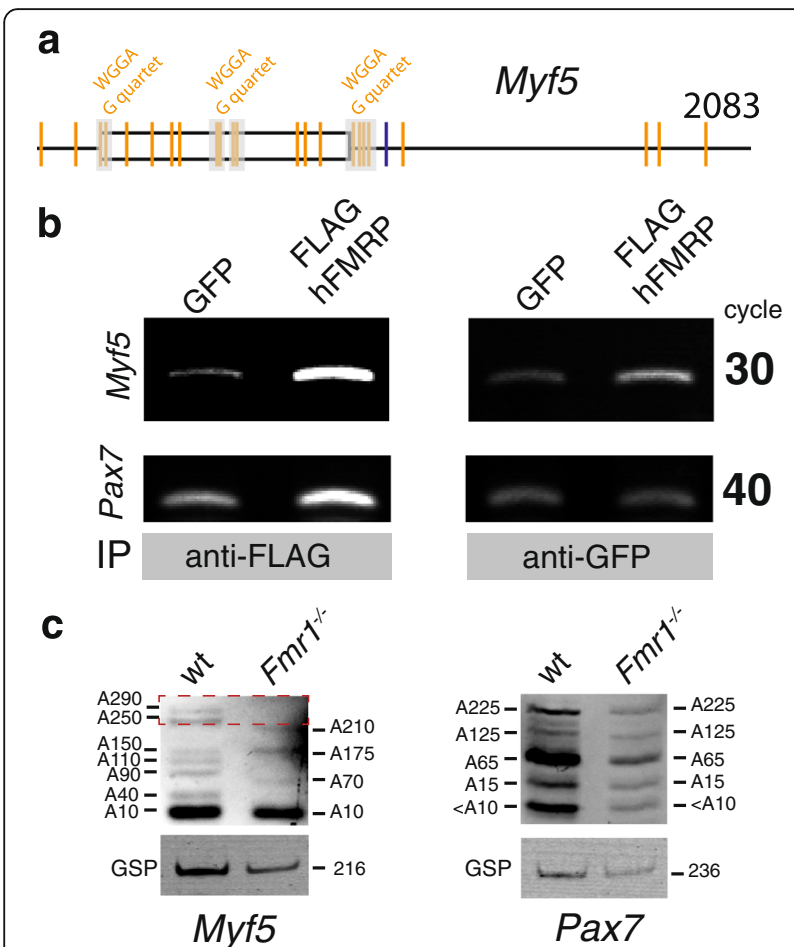

Fig. 5 FMRP binds Myf5 transcripts and affects rates of deadenylation. a Illustration of Myf5 transcripts (Mus musculus) with FMRP binding WGGA (UGGA, AGGA) consensus sites indicated. Consensus sites which are within close proximity to each other and form potential FMRP binding G-quadraplex sites (gray) are indicated. One such G-quadraplex is in proximity to the microRNA-31 (miR-31) binding site on the 3'UTR of Myf5. b Immunoprecipitation (IP) of FMRP-mRNA complexes from C2C12 cells transfected with plasmids encoding GFP and FLAG-hFMRP. Immunoprecipitating antibodies are against FLAG-hFMRP (anti-FLAG, left) and GFP (anti-GFP, right). Amplification of Myf5 (upper panels) and Pax7 (lower panels) transcripts by semi-quantitative RT-PCR are shown, with thermocycle numbers indicated. c Poly(A) tail lengths of Myf5 (left) and Pax7 (right) transcripts amplified from total RNA isolated from 3-day cultures of satellite cells isolated from muscle of adult Pax $3^{\text {GFP/+ }}$ (wild-type, wt) and $\mathrm{Fmrl}^{-1-}$ mice. Estimated polyA lengths are indicated adjacent to each acrylamide gel. Gene-specific primers (GSP) against Myf5 and Pax7, with product size indicated, are shown in the lower panels. A red dotted line indicates Myf5 polyA lengths $<200$ nucleotides present in wild-type but not in $\mathrm{Fmrl}^{-/-}$MuSCs

synthesis required for synaptic development and maturation.

Fragile $\mathrm{X}$ syndrome is also associated with muscle hypotonia (low muscle tone) during infancy [32]. The molecular mechanisms leading to muscle hypotonia are not clear. Given increasing evidence that satellite cells contribute to muscle homeostasis in newborn and young adult mice [33] and our previous observations supporting the cooperation of FMRP with microRNA-31 to regulate satellite cell quiescence and self-renewal [5], we asked whether Fmr1 is required for satellite cell homeostasis. Using Fmr1 ${ }^{-/-}$knockout mice, we demonstrate a role for FMRP in satellite cell activity. First, we show that skeletal muscle of $\mathrm{Fmrl}^{-/-}$knockout mice have approximately half the normal complement of satellite cells. Since numbers of satellite cells are not further reduced after an acute injury, it was difficult to determine whether self-renewal of satellite cells is normal in the absence of FMRP. We therefore used an engraftment assay to determine the fitness of donor muscle stem cells to differentiate and self-renew to repopulate the satellite cell pool. We show that satellite cells without FMRP retain their ability to differentiate, but have reduced capacity to self-renew and repopulate muscle with donorderived satellite cells.

After an acute injury to the skeletal muscle, we also observe delays in regeneration of muscle. These delays may be in part due to a reduced number of satellite cells available to take part in skeletal muscle regeneration, but also in the intrinsic ability of $F m r 1^{-/-}$satellite cells to activate the myogenic program and differentiate to repair muscle. We show that $F m r 1^{-1-}$ satellite cells are defective in two additional properties. First, quiescent $\mathrm{Fmrl}^{-/-}$satellite cells are less able to prime the myogenic program and show reduced levels of $M y f 5$ and $M y o D$ transcripts, which are normally present but translationally silenced. Next, we provide evidence that $F m r 1^{-/-}$satellite cells also take longer to activate the expression of MYF5 and $M Y O D$ protein, which normally occurs within $1 \mathrm{~h}$ after isolation of single EDL myofibers. Second, $\mathrm{Fmrl}^{-/-}$satellite cells have reduced capacity to differentiate, illustrated by delayed expression of MYOD, MYOG and MYH3.

Altogether, we provide evidence that FMRP is required for satellite cells to (a) be primed for rapid activation, (b) efficiently execute the myogenic program to repair muscle after acute injury, and (c) self-renew. Here, we propose a model by which these three qualities of the satellite cell are influenced by the ability of FMRP to bind and regulate the stability and translation of $M y f 5$ transcripts. First, $M y f 5$ transcripts accumulate in the quiescent satellite cell, but remain translationally repressed [5]. How Myf5 transcripts are repressed by the microRNA pathway, but remain stable, remains unclear. Our model by which FMRP directly binds and stabilizes Myf5 transcripts is supported by a reduction of $M y f 5$ transcripts in $\mathrm{Fmr1}^{-/-}$quiescent satellite cells and immunoprecipitation of $M y f 5$ transcripts by FMRP in C2C12 myogenic cells. Second, during satellite cell activation, the continued stability of a pool of Myf5 transcripts, facilitated by FMRP, would ensure rapid entry into the myogenic program and robust differentiation. Third, satellite cell self-renewal activity to repopulate the satellite cell pool after acute injury or after engraftment is also facilitated by FMRP, which is required to re-establish translational repression of $M y f 5$ transcripts to ensure MYF5 protein is not produced when satellite cells reacquire their quiescent state in their normal anatomical satellite position. 
In our model, the bi-functional role of FMRP to repress $M y f 5$ translation during quiescence and permit Myf5 translation during satellite cell activation could also be dependent on FMRP interaction with RISC. This switch is potentially mediated by FMRP phosphorylation, as we have previously proposed [5] in a similar way by which FMRP participates in reversible microRNA silencing observed at dendritic spines of hippocampal neurons [14]. FMRP interaction with other RNA-binding proteins and helicases [34] has also been proposed as mechanisms mediating the bi-functional role of FMRP.

It is unknown if FMRP influences rates of deadenylation of its target transcripts. Given a role for FMRP in reversible microRNA silencing of transcripts in neurons and muscle, we asked whether Myf5 deadenylation rates in activated satellite cells is influenced by the presence or absence of FMRP. We show reduced accumulation of Myf5 transcripts with long poly(A) tails in $\mathrm{Fmrl}^{-/-}$satellite cells, compared to wild-type satellite cells.

While we provide evidence that FMRP binds Myf5 and influences rates of $M y f 5$ deadenylation, it remains likely that FMRP also binds and regulates the stability of other myogenic regulatory factors. While we also observed lower expression levels of MyoD, Myog, and Myh3 transcripts as $\mathrm{Fmrl}^{-/-}$satellite cells differentiate, it remains unclear whether FMRP directly binds these transcripts, or if their lower expression is due in part to reduced expression of upstream factors.

We also note that we observe defective satellite cell properties that lead to reduced differentiation and selfrenewal, but we do not observe a collapse of regeneration or muscle homeostasis. This may be due in part to the redundancy observed between myogenic regulatory factors and the post-transcriptional silencing platforms that regulate their expression. We would expect, for example, that in genetic manipulations eliminating both Fmr1 and tristetraprolin (Ttp), the protein product of which regulates the stability of $M y o D$ to produce a more dramatic phenotype. In addition, while our ex vivo studies demonstrate intrinsic deficiencies in satellite cells, we cannot rule out the possibility that delays in regeneration we have observed are caused by defective cells that are normally required to support regeneration. Our results provide further evidence supporting a role for post-transcriptional silencing platforms by RNA-binding proteins in maintaining stemness properties of adult stem cells.

\section{Conclusions}

In conclusion, we propose a model by which adult stem cells employ translational control of gene expression to prime activation of a differentiation program. In skeletal muscle, priming of the myogenic program is illustrated by accumulation of transcripts required for the myogenic program, including Myf5. In our model, the efficiency of translation of Myf5 is modulated by the activity of FMRP, which directly binds to Myf5 and regulates rates of poly-adenylation to ensure Myf5 stability. Genetic manipulations that eliminate Myf5 result in decreased capacity to prime the myogenic program, slower entry into the myogenic program, and delayed regeneration.

\section{Abbreviations}

ctx: Cardiotoxin; EDL: Extensor digitorum longus; EdU: 5-Ethynyl-2'deoxyuridine; embMHC: Embryonic myosin heavy chain; FMRP: Fragile $X$ mental retardation protein; MuSCs: Muscle stem cells; NMJ: Neuromuscular junction; polyA: Poly-adenine; TA: Tibialis anterior

\section{Acknowledgements}

Not applicable

\section{Funding}

This study is funded by the Canadian Institutes for Health Research (CIHR 286519), the Stem Cell Network, and the Muscular Dystrophy Association (MDA 351259).

\section{Availability of data and materials \\ Not applicable}

\section{Authors' contributions}

CC conceived and designed the study. RF, VZ, JMJ, SJ, and CC performed the experiments and collected the data. KA irradiated the mouse hindlimbs. CC, VZ, and RF wrote the paper. All authors read and approved the final manuscript.

Ethics approval and consent to participate

Not applicable

Consent for publication

Not applicable

\section{Competing interests}

The authors declare that they have no competing interests.

\section{Publisher's Note}

Springer Nature remains neutral with regard to jurisdictional claims in published maps and institutional affiliations.

\section{Author details}

'Lady Davis Institute for Medical Research, Jewish General Hospital, Montreal, Quebec H3T 1E2, Canada. ${ }^{2}$ Department of Human Genetics, McGill University, Montreal, Quebec H3A 1B1, Canada. ${ }^{3}$ Department of Radiation Oncology, Jewish General Hospital, Montreal, Quebec H3T 1E2, Canada. ${ }^{4}$ Current address: Unité Stroma, Inflammation and Tissue Repair, Institut Pasteur, Paris 75724, France.

Received: 9 June 2017 Accepted: 1 September 2017

Published online: 07 September 2017

\section{References}

1. Mauro A: Satellite cell of skeletal muscle fibers. J Biophys Biochem Cytol. 1961;9:493-495

2. Relaix F, Montarras D, Zaffran S, Gayraud-Morel B, Rocancourt D, Tajbakhsh S, Mansouri A, Cumano A, Buckingham M. Pax3 and Pax7 have distinct and overlapping functions in adult muscle progenitor cells. J Cell Biol. 2006;172:91-102.

3. Seale P, Sabourin LA, Girgis-Gabardo A, Mansouri A, Gruss P, Rudnicki MA. Pax7 is required for the specification of myogenic satellite cells. Cell. 2000;102:777-86.

4. Relaix F, Rocancourt D, Mansouri A, Buckingham M. A Pax3/Pax7-dependent population of skeletal muscle progenitor cells. Nature. 2005:435:948-53.

5. Crist CG, Montarras D, Buckingham M. Muscle satellite cells are primed for myogenesis but maintain quiescence with sequestration of Myf5 mRNA targeted by microRNA-31 in mRNP granules. Cell Stem Cell. 2012;11:118-26. 
6. Hausburg MA, Doles JD, Clement SL, Cadwallader AB, Hall MN, Blackshear PJ, Lykke-Andersen J, Olwin BB. Post-transcriptional regulation of satellite cell quiescence by TTP-mediated mRNA decay. elife. 2015;4:e03390.

7. Cheung TH, Quach NL, Charville GW, Liu L, Park L, Edalati A, Yoo B, Hoang $P$, Rando TA. Maintenance of muscle stem-cell quiescence by microRNA489. Nature. 2012:482:524-8.

8. Zismanov V, Chichkov V, Colangelo V, Jamet S, Wang S, Syme A, Koromilas $A E$, Crist C. Phosphorylation of elF2a is a translational control mechanism regulating muscle stem cell quiescence and self-renewal. Cell Stem Cell. 2016;18:79-90

9. Zalfa F, Eleuteri B, Dickson KS, Mercaldo V, De Rubeis S, di Penta A, Tabolacci E, Chiurazzi P, Neri G, Grant SGN, Bagni C. A new function for the fragile $X$ mental retardation protein in regulation of PSD-95 mRNA stability. Nat Neurosci. 2007;10:578-87.

10. Ceman S, O'Donnell WT, Reed M, Patton S, Pohl J, Warren ST. Phosphorylation influences the translation state of FMRP-associated polyribosomes. Hum Mol Genet. 2003;12:3295-305.

11. Caudy AA, Myers M, Hannon GJ, Hammond SM. Fragile X-related protein and VIG associate with the RNA interference machinery. Genes Dev. 2002;16:2491-6.

12. Ishizuka A, Siomi MC, Siomi H. A Drosophila fragile $X$ protein interacts with components of RNAi and ribosomal proteins. Genes Dev. 2002;16:2497-508.

13. Jin P, Zarnescu DC, Ceman S, Nakamoto M, Mowrey J, Jongens TA, Nelson DL, Moses K, Warren ST. Biochemical and genetic interaction between the fragile $X$ mental retardation protein and the microRNA pathway. Nat Neurosci. 2004;7:113-7

14. Muddashetty RS, Nalavadi VC, Gross C, Yao X, Xing L, Laur O, Warren ST, Bassell GJ. Reversible inhibition of PSD-95 mRNA translation by miR-125a, FMRP phosphorylation, and mGluR signaling. Mol Cell. 2011;42:673-88.

15. Fmr1 knockout mice: a model to study fragile $X$ mental retardation. The Dutch-Belgian Fragile X Consortium. Cell. 1994;78:23-33.

16. Montarras D, Buckingham M. Direct isolation of satellite cells for skeletal muscle regeneration. Science. 2005;309:2064-7.

17. Yamaguchi M, Watanabe $Y$, Ohtani T, Uezumi A, Mikami N, Nakamura M Sato T, Ikawa M, Hoshino M, Tsuchida K, Miyagoe-Suzuki Y, Tsujikawa K, Takeda S, Yamamoto H, Fukada S-I. Calcitonin receptor signaling inhibits muscle stem cells from escaping the quiescent state and the niche CellReports. 2015;13:302-14.

18. Matsuda T, Cepko CL. Electroporation and RNA interference in the rodent retina in vivo and in vitro. Proc Natl Acad Sci U S A. 2004;101:16-22.

19. Ascano M, Mukherjee N, Bandaru P, Miller JB, Nusbaum JD, Corcoran DL, Langlois C, Munschauer M, Dewell S, Hafner M, Williams Z, Ohler U, Tuschl T. FMRP targets distinct $m R N A$ sequence elements to regulate protein expression. Nature. 2012;492:382-6.

20. Peritz T, Zeng F, Kannanayakal TJ, Kilk K, Eiríksdóttir E, Langel U, Eberwine J. Immunoprecipitation of mRNA-protein complexes. Nat Protoc. 2006;1:577-80.

21. Kelly AM. Perisynaptic satellite cells in the developing and mature rat soleus muscle. Anat Rec. 1978;190:891-903.

22. Wokke JH, Van den Oord CJ, Leppink GJ, Jennekens FG. Perisynaptic satellite cells in human external intercostal muscle: a quantitative and qualitative study. Anat Rec. 1989;223:174-80.

23. Schultz E. Changes in the satellite cells of growing muscle following denervation. Anat Rec. 1978;190:299-311.

24. Zhang YQ, Bailey AM, Matthies HJ, Renden RB, Smith MA, Speese SD, Rubin GM, Broadie K. Drosophila fragile $X$-related gene regulates the MAP1B homolog Futsch to control synaptic structure and function. Cell. 2001;107:591-603.

25. Couteaux R, Mira JC, d'Albis A. Regeneration of muscles after cardiotoxin injury. I. Cytological aspects. Biol Cell. 1988:62:171-82.

26. Darnell JC, Jensen KB, Jin P, Brown V, Warren ST, Darnell RB. Fragile $X$ mental retardation protein targets $\mathrm{G}$ quartet mRNAs important for neuronal function. Cell. 2001;107:489-99.

27. Anderson BR, Chopra P, Suhl JA, Warren ST, Bassell GJ. Identification of consensus binding sites clarifies FMRP binding determinants. Nucleic Acids Res. 2016;44:6649-59.

28. Fabian MR, Mathonnet G, Sundermeier T, Mathys H, Zipprich JT, Svitkin $\mathrm{W}$, Rivas F, Jinek M, Wohlschlegel J, Doudna JA, Chen C-YA, Shyu A-B, Yates JR, Hannon GJ, Filipowicz W, Duchaine TF, Sonenberg N. Mammalian miRNA RISC recruits CAF1 and PABP to affect PABP-dependent deadenylation. Mol Cell. 2009;35:868-80.
29. Fabian MR, Frank F, Rouya C, Siddiqui N, Lai WS, Karetnikov A, Blackshear PJ, Nagar B, Sonenberg N. Structural basis for the recruitment of the human CCR4-NOT deadenylase complex by tristetraprolin. Nat Struct Mol Biol. 2013:20:735-9.

30. Leung C, Jia Z. Mouse genetic models of human brain disorders. Front Genet. 2016;7:189-20.

31. Verkerk AJ, Pieretti M, Sutcliffe JS, Fu YH, Kuhl DP, Pizzuti A, Reiner O, Richards S, Victoria MF, Zhang FP. Identification of a gene (FMR-1) containing a CGG repeat coincident with a breakpoint cluster region exhibiting length variation in fragile X syndrome. Cell. 1991;65:905-14.

32. Wisniewski KE, Segan SM, Miezejeski CM, Sersen EA, Rudelli RD. The Fra $(X)$ syndrome: neurological, electrophysiological, and neuropathological abnormalities. Am J Med Genet. 1991;38:476-80.

33. Pawlikowski B, Pulliam C, Betta ND, Kardon G, Olwin BB. Pervasive satellite cell contribution to uninjured adult muscle fibers. Skelet Muscle. 2015:5:42

34. Kenny P, Ceman S. RNA secondary structure modulates FMRP's bi-functional role in the microRNA pathway. IJMS. 2016;17:985-13.

\section{Submit your next manuscript to BioMed Central and we will help you at every step:}

- We accept pre-submission inquiries

- Our selector tool helps you to find the most relevant journal

- We provide round the clock customer support

- Convenient online submission

- Thorough peer review

- Inclusion in PubMed and all major indexing services

- Maximum visibility for your research

Submit your manuscript at www.biomedcentral.com/submit
Ciomed Central 